\title{
CRYPTOGENIC SENSORY POLYNEUROPATHY
}

\author{
Mamatha Pasnoor, MD [Assistant Professor], \\ Department of Neurology, University of Kansas Medical Center, Kansas City, KS
}

Mazen M. Dimachkie, M.D. [Professor], and Neurology, Director, Neuromuscular Section, Department of Neurology, University of Kansas Medical Center, Kansas City, KS

Richard J. Barohn, MD [Professor and Chairman] Department of Neurology, University of Kansas Medical Center, Kansas City, KS

\begin{abstract}
Chronic sensory or sensorimotor polyneuropathy is a common cause for referral to neurologists. Despite extensive diagnostic testing, up to one-third of these patients remain without a known cause. They are referred to as having cryptogenic sensory peripheral neuropathy (CSPN). The age of onset is variable but usually in the sixth to seventh decade of life, affecting men and women equally. CSPN symptoms progress slowly, most patients present with distal leg paresthesias or pain that progressed over years to involve the hands. On examination, there may be additional mild toe flexion and extension weakness. Electrophysiologic testing and histology reveals axonal neuropathy. Prognosis is usually favorable as most patients maintain independent ambulation. Besides patient education and reassurance, management is focused on pharmacotherapy of neuropathic pain (see Treatment of Painful Peripheral Neuropathy chapter) and physical therapy for balance training and occasionally assistive devices.
\end{abstract}

\section{Introduction}

Acquired chronic sensory and sensorimotor polyneuropathies are common in middle and late adulthood, with an estimated prevalence of more than $3 \% .^{1}$ The majority of acquired neuropathies are secondary to readily identifiable causes, such as diabetes, alcohol abuse, or iatrogenic causes, specifically medications. However, once known etiologies are excluded, a sizable minority of acquired neuropathies remains idiopathic, and we refer to them herein as CSPN. Prior reports describing CSPN have used other terms such as idiopathic neuropathy or small fiber sensory peripheral neuropathy but we prefer CSPN. The diagnostic criteria for CSPN have been established by Wolfe et al and are used by many physicians (table 3 ). ${ }^{2}$ In earlier series, the cryptogenic group was thought to comprise as much as $50-70 \%$ of

(C) 2013 Elsevier Inc. All rights reserved.

Correspondence: Mamatha Pasnoor, MD, University of Kansas Medical Center, 3599 Rainbow Blvd, Mail-stop 2012, Kansas City, KS 66160, Phone: 913-588-0668, Fax: 913-588-0673, mpasnoor@kumc.edu.

Publisher's Disclaimer: This is a PDF file of an unedited manuscript that has been accepted for publication. As a service to our customers we are providing this early version of the manuscript. The manuscript will undergo copyediting, typesetting, and review of the resulting proof before it is published in its final citable form. Please note that during the production process errors may be discovered which could affect the content, and all legal disclaimers that apply to the journal pertain. 
polyneuropathy (PN) cases (table 1). ${ }^{3-5}$ These studies, however were largely based on younger groups of inpatients, many of whom presented with severe weakness resembling acute or chronic inflammatory demyelinating PN. ${ }^{3}$ Later studies have revised the frequency of CSPN downward to $10-35 \%$ with most estimates clustered in the $10-25 \%$ range. . $^{2,6-11}$ One recent study that included testing for impaired glucose tolerance and celiac disease in patients with abnormal skin biopsy findings, found $50 \%$ to be idiopathic. ${ }^{12}$ Our retrospective review looking at one North America site and 2 South America site databases (NASA) showed that CSPN represented (approximately 25\%) of all referred PN patients(table 2). ${ }^{13,55}$ Likely reasons for the declining percentage include improvement in recognition of hereditary neuropathies, and in the identification of immune-mediated neuropathies, as well as the investigative plans becoming more sophisticated and modern pattern-based diagnostic approaches. $9,10,14$

For a relatively common clinical problem, there are surprisingly few detailed reports of CSPN. The types of PN patients included differ between studies, making generalization, somewhat difficult. Earlier studies did not provide detailed laboratory and electrophysiologic data. Nevertheless, the bulk of information available suggests that CSPN is predominantly sensory distal dying back axonopathy that progresses little or slowly over time.

\section{Diagnostic Criteria}

CSPN is in essence a diagnosis of exclusion, established after a careful medical, family and social history, neurologic examination and directed laboratory testing. England et al recently reported the recommendations of the AAN Practice Parameter Committee for the evaluation of distal symmetric polyneuropathy (DSP). ${ }^{15,16}$ Tests with the highest yield of abnormality in polyneuropathy evaluation are blood glucose, serum B12 with metabolites (methylmalonic acid with or without homocysteine), and serum protein immunofixation electrophoresis. The level of evidence in the literature allowed for the highest level of recommendation being class $\mathrm{C}$. If routine testing of blood glucose is negative for diabetes mellitus, testing for impaired glucose tolerance may be considered. Nerve conduction studies are indicated to define whether the neuropathy is axonal or demyelinating, focal or generalized and hereditary or acquired. Skin biopsy also received a level $\mathrm{C}$ recommendation as having a role in the evaluation of DSP, particularly small fiber sensory neuropathy (SFSN). The purpose of such a clinical and laboratory evaluation is to rule out identifiable causes of neuropathy such as diabetes, chronic alcoholism, metabolic disturbances, endocrine abnormalities, connective tissue diseases, malignancy or amyloidosis, HIV or other infections, pertinent toxic or pharmacologic exposures, and hereditary factors. Genetic testing is recommended for the accurate diagnosis and classification of hereditary neuropathies and it may be considered in patients with cryptogenic polyneuropathy who exhibit a hereditary neuropathy phenotype by virtue of family history and long-standing high arches or hammer toes. ${ }^{15,16}$ The literature is inconclusive about the utility of nerve biopsy in the evaluation of DSP. 


\section{Clinical Features}

The onset of idiopathic polyneuropathies occurs mainly in the sixth and seventh decades of life. The mean age of patients in published series ranges from 51 to 63 years of age. ${ }^{2,6,12,17-20}$ There appears to be no difference in the age of onset between sensorimotor and sensory groups. CSPN is usually diagnosed on the basis of pain, numbness and /or tingling in the distal extremities without symptoms of weakness. Sensory symptoms had to occur in a roughly symmetrical pattern in the distal lower extremities or upper extremities or both and evolve over weeks to months. On examination, patients have to demonstrate distal sensory deficits not confined to an individual peripheral nerve. Slight distal weakness, Medical Research Council (MRC) grade 4 or greater in foot or hand intrinsic muscles is permitted as long as motor symptoms are not the presenting complaint. Series of patients with idiopathic sensorimotor neuropathies or mixed groups report a greater number of men than women, although it is unclear if this truly reflects differences in disease prevalence or referral bias. ${ }^{6,10,11,20,21}$ In series of sensory-only or small fiber neuropathy patients, there appear to be equal numbers of men and women represented, and in 1 study of small fiber neuropathy, half of whom were idiopathic, there was a greater number of women than men. ${ }^{2,12}$ Other studies of painful sensory neuropathy also failed to find a male predominance. ${ }^{17,22}$

In patients with sensorimotor idiopathic neuropathy pain is reported in $27 \%$ to $42 \%$ of patients, sensory loss in $65 \%$, paresthesias in $33 \%$ to $68 \%$, weakness in $26 \%$ to $82 \%$, and difficulty with balance in $33 \%{ }^{6,10,11,19,20}$ In series of patients with sensory-only neuropathy or small fiber neuropathy, the most common symptoms are pain in 54\% to $100 \%$ of patients, sensory loss in $86 \%$, and paresthesias in $86 \%$ to $100 \%{ }^{2,10,18,19,22}$ In both groups, upper extremity symptoms are usually preceded by lower extremity involvement. ${ }^{2,10}$ Worsening of sensory symptoms with heat exposure, activity, or fatigue is commonly reported in patients. ${ }^{22}$ Approximately one-third to one-half of patients will have symptoms confined to their lower extremities. ${ }^{10,22}$ The average time or symptoms to spread to the upper extremities appears to be about 5 years. ${ }^{10}$ It is rare for patients to report symptoms restricted to the upper extremities. ${ }^{21}$ Autonomic and cranial nerve findings are rare. ${ }^{22}$

On examination of CSPN patients, the most common findings are reduced or absent lower extremity light touch in 59\% to $91 \%$, pinprick in $53 \%$ to $95 \%$, vibratory sense in $80 \%$ to $100 \%$, while proprioception is usually spared being abnormal in $9 \%$ to $18 \%$. Sensory abnormalities in the hands occur in $36 \%$ of patients. On motor examination, there is distal lower extremity weakness primarily affecting toe flexion and extension in 39\% to $100 \%$ of patients and intrinsic hand muscle weakness in $10 \%$. Reduced or absent ankle jerk reflexes are reported in $78 \%$ to $100 \%$ of patients. ${ }^{2,6,10,11,20}$ Distal atrophy may be present and as expected foot deformities may develop after a decade from neuropathy onset.

\section{Diagnosis}

\section{Laboratory}

The basic and highest yield laboratory studies in DSP include blood glucose, vitamin B12 level with metabolites (methylmalonic acid and homocysteine), and serum protein 
immunofixation electrophoresis. ${ }^{15}$ If those studies are normal, oral glucose tolerance testing should be completed, especially if the clinical presentation includes pain. ${ }^{15,16}$ Other laboratory studies also recommended in routine lab testing include a basic metabolic panel, complete blood count, erythrocyte sedimentation rate, and antinuclear antigen,. The following studies are rarely useful in CSPN cases but should also be considered in the appropriate clinical scenario: urine protein electrophoresis, antineutrophil cytoplasmic antigen (ANCA) antibodies, serologies for IgG and IgA antigliadin antibodies, IgA antitransglutaminase antibodies, human immunodeficiency virus (HIV) antibody, Lyme antibodies and western blot, heavy metal levels, and paraneoplastic and other antibody assays.

Testing for vitamin B12 deficiency is relatively high-yield and should include testing levels of cobalamin metabolites, methylmalonic acid, and homocysteine. In 2 series of patients with polyneuropathy, between $2.2 \%$ and $8 \%$ of patients had B12 deficiency, and in $5 \%$ to $10 \%$ of patients with serum B12 levels in the low to normal range, methylmalonic acid and homocysteine levels were elevated. ${ }^{27-30}$

The incidence of monoclonal proteins in chronic idiopathic polyneuropathy is as high as $10 \%$, representing a monoclonal gammopathy of uncertain significance in most patients. ${ }^{25-28}$ Patients with an IgM monoclonal gammopathy commonly have specific antibody binding against peripheral nerve antigens, implicating an autoantibody pathogenesis of associated neuropathies. ${ }^{31}$ However, polyneuropathy patients with monoclonal gammopathy of uncertain significance are difficult to distinguish on clinical and electrophysiological grounds from idiopathic polyneuropathy patients without a paraprotein, raising questions about the pathogenic role of the immunoglobulin and whether the monoclonal gammopathy of uncertain significance, particularly $\operatorname{IgG}$ and $\operatorname{IgA}$, is a coincidental discovery. ${ }^{32,33}$ Incidental paraproteins are found in up to $3 \%$ of the general elderly population, the age group with the highest prevalence of polyneuropathies. ${ }^{27}$

Data on anti-sulfatide antibodies has produced conflicting results that renders testing of limited value in CSPN. In earlier studies of idiopathic sensory-predominant polyneuropathy, approximately one quarter of patients selected from case records or a clinic population had anti-sulfatide antibodies. ${ }^{34,35}$ However, other series have found anti-sulfatide antibodies in none or only a small percentage of the patients with idiopathic polyneuropathy. $2,10,17,18,32$

\section{Lumbar puncture}

CSF analysis is not essential to the diagnosis of CSPN. In earlier series, it is largely unremarkable. The mean CSF protein from 73 unclassified patients in a series was $43 \mathrm{mg}$ / dl. ${ }^{10} \mathrm{CSF}$ was normal in 4 of 5 patients from another series. ${ }^{2}$ In a 2001 series of 8 patients who had lumbar puncture for idiopathic neuropathy, none had elevated protein. ${ }^{11}$ Although typically normal in idiopathic polyneuropathies, CSF analysis should be considered in patients with rapidly progressive symptoms, or of if there is clinical suspicion of sensory chronic inflammatory demyelinating polyneuropathy (CIDP) as also suggested by nerve conduction studies. 


\section{Genetic Testing}

Genetic testing is not generally indicated in CSPN. Patients with unexplained family history of neuropathy (such as unrelated to diabetes), distal weakness as the presenting complaint, life-long foot deformities preceding the onset of numbness or markedly demyelinating electrophysiology should be suspected to have a genetic etiology for their neuropathy. In this small subgroup of CSPN, genetic testing may be appropriate. Otherwise, it should be reserved for hereditary peripheral neuropathies, such as Charcot-Marie-Tooth disease, in which genetic mutations are detectable in $54 \%$ to $100 \% .{ }^{36-39}$ The role of genetic testing in CSPN patients without the classic phenotype is unknown. ${ }^{15}$

\section{Electrophysiology}

\section{Nerve Conduction Studies}

Besides assessing severity, nerve conduction studies are indicated to define whether a neuropathy is axonal or demyelinating and focal or generalized. CSPN is almost exclusively an axonal neuropathy as none of the CSPN patients in Wolfe's series had demyelinating features on nerve conduction studies (NCS) Wolfe 1999). NCS abnormalities should be expected in the vast majority of CSPN patients, $77-97 \% .2,6,10$ Sural sensory nerve action potential testing has the greatest yield, being abnormal in $69 \%$ of the CSPN patients and at least $83 \%$ in the sensorimotor PN patients. ${ }^{10}$ Sensory and motor NCS abnormalities typically consist of reduced amplitudes with normal or minimal distal latency and conduction velocity changes. However, Gorson and Ropper reported normal NCS in $45 \%$ of patients presenting with burning, painful paresthesias from presumed idiopathic distal smallfiber neuropathy. ${ }^{22}$

\section{Electromyogram}

A majority of patients demonstrate abnormalities on needle electromyography, even if there is no evidence of motor nerve involvement by history or examination (Wolfe 1999, Gorson 1995,Schect 1996). ${ }^{2,22,40}$ In a large study, 70\% of patients had an abnormal electromyography. ${ }^{2}$ Distal leg fibrillations potentials were present in $42 \%$ and neurogenic motor unit potentials in $63 \%$. Electromyography abnormalities have also been found in smaller series of patients with pure sensory presentations. ${ }^{22}$ Therefore, it is not uncommon for cryptogenic PN patients who only have sensory symptoms and signs to demonstrate subclinical motor involvement on electrophysiologic studies. Absence of the sural nerve sensory nerve action potential or the presence of spontaneous muscle fiber activity (ie, fibrillation potentials, positive sharp waves, or complex repetitive discharges) in the anterior tibial muscle were found in $72 \%$ of young and old CSPN patients, Chronic idiopathic axonal polyneuropathy and successful aging of the peripheral nervous system in elderly people Arch Neurol. ${ }^{41}$ In comparison, only $2 \%$ of the young and old control group $(n=49)$ had such an abnormality; one had absent sural nerve action potential and another mild positive sharp waves of the anterior tibial muscle. 


\section{Quantitative sensory testing}

Quantitative sensory testing has been applied in several studies of idiopathic polyneuropathies. By measuring thermal thresholds, quantitative sensory testing has a potential advantage over routine nerve conduction studies in the assessment of small sensory fibers, the fiber population predominantly affected by many idiopathic polyneuropathies. Quantitative sensory testing commonly demonstrates abnormalities in patients with symptoms of neuropathy, and it was slightly more sensitive than nerve conduction studies in 1 large study. ${ }^{2,18,42}$ However, no concordance was observed between quantitative sensory testing and intraepidermal nerve fiber density measurements in another study. ${ }^{18}$ Quantitative sensory testing also requires subjective patient input and may reflect abnormalities outside of the peripheral nervous system. ${ }^{43-45}$ Despite good test-retest and interobserver reliability following standardized training, QST results should not be the sole diagnostic criteria since malingering and other nonorganic factors can influence the test results. ${ }^{46,47}$

\section{Autonomic testing}

Autonomic testing should be considered for patients with polyneuropathy, particularly if there is clinical evidence for autonomic neuropathy or distal small fiber sensory polyneuropathy. Measurements of heart rate variability and quantitative sudomotor axon reflex testing (QSART) have been shown to be sensitive and specific for polyneuropathy, especially when used in combination. ${ }^{16}$

\section{Pathology}

Nerve biopsy generally confirms the axonal nature of idiopathic polyneuropathies. All 31 nerve specimens from a large series showed axonal degeneration. ${ }^{10}$ There was no evidence of demyelination, inflammation, vasculitis, or amyloidosis. Of 14 sural nerve biopsies from another large series, 13 demonstrated typical features of axonal degeneration. ${ }^{2}$ These studies suggest that sural nerve biopsy is rarely helpful in patients with idiopathic sensory neuropathies. Although biopsy findings typically demonstrate evidence of axonal damage, a recent series reported 8 patients with cryptogenic sensory neuropathy who had pathologic findings consistent with chronic myelinopathy. ${ }^{48}$ Nerve biopsy should be considered, however, when there is clinical suspicion for certain types of neuropathies, including amyloid neuropathy, mononeuritis multiplex due to vasculitis, or atypical chronic inflammatory demyelinating polyneuropathy (CIDP). ${ }^{16}$

Skin biopsy for measurement of intraepidermal nerve fiber (IENF) density should be considered in the workup of distal sensory neuropathy, particularly in cases of small fiber sensory neuropathy. The most commonly used technique involves a $3 \mathrm{~mm}$ punch biopsy of skin from the leg, which is sectioned and examined using immunostaining techniques for anti-protein-gene-product 9.5 (PGP 9.5) antibodies in order to count the IENF density. ${ }^{15}$ Skin biopsy for IENF density has been shown to be reliable and valid in patients with distal sensory neuropathy, particularly in patients with small fiber neuropathy, with a reported diagnostic efficiency of $88 \% .{ }^{49,50}$ It is more sensitive than quantitative sensory testing or sudomotor autonomic testing, and 1 series demonstrated reduced IENF density in three quarters of patients with painful sensory symptoms and normal nerve conduction studies. ${ }^{18}$ 
In another study, IENF on skin biopsy was abnormal in $39 \%$ of 158 patients with clinically suspected small fiber neuropathy, of which $50 \%$ were of idiopathic etiology. ${ }^{12}$ High concordance has been reported between reduced IENF density and loss of pinprick on clinical exam. ${ }^{51}$ Skin biopsy for IENF density has also been shown to be more sensitive for small fiber sensory neuropathy than sural nerve biopsy, but it is typically normal in demyelinating neuropathies. ${ }^{52}$ Guidelines from both the American Academy of Neurology (AAN) and European Federation of Neurological Societies (EFNS) have recommended that skin biopsy for determination of IENF density is a valid and reliable method for diagnosis of distal sensory neuropathy, particularly small fiber sensory neuropathy. ${ }^{15,53}$ IENF density in CSPN patients may not be significantly different from diabetic neuropathy patients. ${ }^{54}$

\section{Clinical Course}

Consistent with other prior reports, fewer than 10\% of CSPN patient's in Wolfe's series showed progression on follow-up. All remained ambulatory during the mean follow-up period of 8 months. ${ }^{7,10,17,32}$ The majority of patients with idiopathic sensory and sensorimotor polyneuropathies follow a stable to slowly progressive course over years. $2,6,7,10,11,17,19,22$ A plateau phase of relative stability is particularly characteristic of pure sensory polyneuropathies. ${ }^{10}$ Independent ambulation is maintained in nearly all patients, even after follow-up intervals stretching as long as 9 years. ${ }^{21}$ However, walking canes and ankle bracing may be required over time in the sensorimotor population. ${ }^{17}$ The need for assistive devices in patients with pure sensory presentations appears to be uncommon. In the series of Vranken and colleagues, 35/127 patients used walking aids such as a cane, adjusted shoes, ankle-foot orthoses, or a wheeled walker. ${ }^{41}$ They also found that chronic idiopathic axonal polyneuropathy appears to have a greater influence on the daily life of non retired patients with onset before the age of 65 years.

\section{Yield of Reevaluations}

There is controversy as to whether reevaluation of CSPN patients uncovers a cause in a sizable number of patients. Dyck et al ${ }^{14}$ found that intensive evaluation of referred unclassified neuropathies yielded an identifiable cause in $76 \%$. Grahmann et $\mathrm{al}^{7}$ found that only $17.5 \%$ of outpatients remained unclassified after an initial evaluation. Reevaluation on an outpatient basis further classified 12 of 41 patients (29\%) initially diagnosed as CSPN. McLeod reported an etiology over time in 17 of 47 unclassified patients (36\%). ${ }^{6}$ Notermans et $\mathrm{al}^{10}$ determined an etiology in only 4 of 75 patients despite a follow-up period of over 5 years. Repeating laboratory studies on a routine basis, including serum immunoelectrophoresis, were not informative. Differences in referral populations and in the breadth of the preliminary workup are the most obvious explanations for these contrasting yields on reevalutations. We therefore do not recommend repeat testing in CSPN. However, all patients with suspected CSPN should undergo a 2-hour glucose tolerance test even in the setting of a normal fasting blood glucose and normal hemoglobin A1c, particularly those experiencing pain.

Hereditary neuropathies may account for a large number of undiagnosed PN. McLeod, Notermans, and Dyck found evidence of hereditary process in $42 \%$ of referred patients with 
idiopathic PN. 6,14,17,32 In approximately $30 \%$ of these cases inheritance was not revealed by review of family history and medical records and was only identified after direct examination and electrophysiologic testing of relatives. In Wolfe's series, 29.9\% were hereditary and in the NASA series this represented $27 \%$ of North American cases. ${ }^{55}$ Hereditary motor and sensory neuropathy (HMSN) type 2 should be considered when motor symptoms predominate and skeletal deformities are present. ${ }^{56}$ Muscle cramping in the legs and feet and the absence of paresthesias may favor a hereditary neuropathy over other etiologies. ${ }^{14}$ Onset late in life does not exclude HMSN, as it is estimated that $15 \%$ of patients with autosomal dominant HMSN type 2 present after the age of 50. Alcohol abuse may explain other cases. ${ }^{6,17,32}$

\section{Management}

Treatment of neuropathic pain is the primary focus of management for most CSPN patients. However, treatment of painful paresthesias and dysesthesias is rarely mentioned in prior series of CSPN. Most treatment neuropathic pain studies have focused on painful diabetic and HIV-associated neuropathies, and postherpetic and trigeminal neuralgias. Medications studied for other forms of painful PN are borrowed and applied to the idiopathic group with some success. We performed a retrospective chart review of 143 patients who were started on pregabalin or duloxetine for neuropathic pain. ${ }^{57} \mathrm{We}$ found no statistically significant difference in the proportion of patients that reported improvement in pain or adverse events with pregabalin (33\% and $30 \%$ ) and duloxetine (21\% and $38 \%$ ) respectively. Therefore, our limited data suggests that both pregabalin and duloxetine are probably effective for neuropathic pain including that due to CSPN.

A number of review articles have stratified medications for painful polyneuropathy into first-, second-, and third-line therapies based on the evidence supporting their benefit and potential risks and side effects. ${ }^{58-61}$ All agree that first-line therapies for peripheral neuropathic pain include tricyclic antidepressants and calcium channel alpha2-delta ligands (gabapentin and pregabalin). Topical lidocaine, available in patch and gel forms, is considered first- or second-line therapy for localized peripheral neuropathic pain, with the greatest evidence for postherpetic neuralgia. Antidepressants with dual serotonin and norepinephrine reuptake activity (duloxetine and venlafaxine) are first-line therapy in some reviews and second-line in others. Opioid medications and tramadol have been demonstrated to have good efficacy for neuropathic pain, particularly in combination with gabapentin, but most authors consider these agents to be second- or third-line therapies based on concern for side effects and dependence. ${ }^{62}$ Other medications that are typically classified as third- or fourth-line therapies with inconsistent results for efficacy include other antidepressants (selective serotonin reuptake inhibitors), other antiepileptics (carbamazepine, oxcarbamazepine, lamotrigine, valproate, topiramate), topical capsaicin, cannabinoids, mexiletine, clonidine, and N-methyl-D-aspartate receptor antagonists (memantine, dextromethorphan). The American Academy of Neurology recently puslished evidencebased guidelines on the treatment of painful diabetic neuropathy. ${ }^{63}$ For further details, the reader is referred to the chapter on Treatment of Painful Peripheral Neuropathy in this issue. There is limited data to support the empiric use of immunosuppressive agents in patients with predominantly sensory idiopathic polyneuropathies. Some studies showed use of 
immunosuppressive agents in idiopathic axonal sensorimotor PN. Intravenous gammaglobulin produced nearly complete resolution of burning pain in three patients with small-fiber neuropathy who were refractory to other agents. ${ }^{22}$ Two patients required repeat infusions to control their symptoms. Prednisone and methotrexate have been advocated to improve sensory and motor function in a preliminary report of 13 patients with idiopathic axonal neuropathy. ${ }^{64}$ However, 9 of these patients had asymmetric sensory or motor deficits, 5 had elevated ANA titers, and 7 had perivascular inflammation on muscle or nerve biopsy, all suggesting an immune-mediated process. ${ }^{64}$ There have been individual reports of improvement of pain or motor function with intravenous immune globulin or other immunosuppressants, but evidence is limited and empiric treatment without known etiology is generally not recommended, particularly if the course is benign. ${ }^{22,65}$ Arguments against use of these agents include the potential for serious adverse events, the significant cost, the lack of concrete evidence for an autoimmune or inflammatory etiology for the PN, and the relatively favorable long-term prognosis. Therefore, we do not recommend immunosuppressive therapy on CSPN.

As noted by Vrancken and colleagues, up to $79 \%$ to $18 \%$ of CSPN cases complain of gait instability or loss of finger dexterity, respectively. ${ }^{41}$ Falls in this aged group carries major risks related to long bone and hip fractures. In a cross-sectional study of 82 subjects aged 50 to 85 with clinical and electrodiagnostic evidence of PN, $48.8 \%, 34.1 \%$ and $22.0 \%$ of subjects reported over the last 2 years a history of at least one fall, multiple falls, and injurious falls, respectively. ${ }^{66}$ Hence, rehabilitative measures are an important consideration including hand occupational therapy in some cases. Despite limited data, we also recommend gait training physical therapy for patient with impaired balance and in those experiencing falls or near falling. A recent literature review supports the safety and efficacy of strength and balance training in reducing falls and improving lower extremity strength and balance for individuals with peripheral neuropathy. ${ }^{67}$

Patient education plays an important role in the management of idiopathic polyneuropathy. Patients are often anxious about their future, with many having been told by other physicians that their prognosis is bleak and that they will become physically incapacitated over time. The clinician can be a source of reassurance by referring to available literature stating that the vast majority of patients remain stable or progress slowly over time, suffer limited motor disability, and have a relatively favorable long-term prognosis. Simply relaying this natural history to patients can provide considerable emotional and even physical comfort.

\section{References}

1. Beghi E, Monticelli ML. Chronic symmetric symptomatic polyneuropathy in the elderly: a field screening investigation in two Italian regions. I. Prevalence and general characteristics of the sample. Italian General Practitioner Study Group (IGPSG). Neurology. 1995; 45(10):1832-1836. [PubMed: 7477977]

2. Wolfe GI, Baker NS, Amato AA, et al. Chronic cryptogenic sensory polyneuropathy: clinical and laboratory characteristics. Arch Neurol. 1999; 56(5):540-547. [PubMed: 10328248]

3. Matthews WB. Cryptogenic polyneuritis. Proc R Soc Med. 1952; 45:667-669.

4. Rose FC. Peripheral neuropathy. Proc R Soc Med. 1960:5351-5353. 
5. Fagius J. Chronic cryptogenic polyneuropathy: the search for a cause. Acta Neurol Scand. 1983:67173-67180.

6. McLeod JG, Tuck RR, Pollard JD, Cameron J, Walsh JC. Chronic polyneuropathy of undetermined cause. J Neurol Neurosurg Psychiatry. 1984:47530-47535.

7. Grahmann F, Winterholler M, Neundörfer B. Cryptogenic polyneuropathies: an out-patient followup study. Acta Neurol Scand. 1991:84221-84225.

8. Hopf HC, Althaus HH, Vogel P. An evaluation of the course of peripheral neuropathies based on clinical and neurographical re-examinations. Eur Neurol. 1973:990-1104.

9. Corvisier N, Vallat JM, Hugon J, Lubeau M, Dumas M. Les neuropathies de cause indéterminée. Rev Neurol (Paris). 1987:143279-143283.

10. Notermans NC, Wokke JHJ, Franssen H, et al. Chronic idiopathic polyneuropathy presenting in middle or old age: a clinical and electrophysiological study of 75 patients. J Neurol Neurosurg Psychiatry. 1993:561066-561071.

11. Jann S, Beretta S, Bramerio M, Defanti CA. Prospective follow-up study of chronic polyneuropathy of undetermined cause. Muscle Nerve. 2001; 24(9):1197-1201. [PubMed: 11494273]

12. De Sousa EA, Hays AP, Chin RL, Sander HW, Brannagan TH 3rd. Characteristics of patients with sensory neuropathy diagnosed with abnormal small nerve fibres on skin biopsy. J Neurol Neurosurg Psychiatry. 2006; 77(8):983-985. [PubMed: 16844956]

13. Khan S, Wolfe G, Nascimento O, Pasnoor M, et al. North American and South America (NA-SA) Neuropathy project (abstract). Neurology. 2006; 66:A84.

14. Dyck PJ, Oviatt KF, Lambert EH. Intensive evaluation of referred unclassified neuropathies yields improved diagnosis. Ann Neurol. 1981; 10(3):222-226. [PubMed: 7294727]

15. England JD, Gronseth GS, Franklin G, et al. Practice Parameter: evaluation of distal symmetric polyneuropathy: role of laboratory and genetic testing (an evidence-based review). Report of the American Academy of Neurology, American Association of Neuromuscular and Electrodiagnostic Medicine, and American Academy of Physical Medicine and Rehabilitation. Neurology. 2009a; 72(2):185-192. [PubMed: 19056666]

16. England JD, Gronseth GS, Franklin G, et al. Practice Parameter: evaluation of distal symmetric polyneuropathy: role of autonomic testing, nerve biopsy, and skin biopsy (an evidence-based review). Report of the American Academy of Neurology, American Association of Neuromuscular and Electrodiagnostic Medicine, and American Academy of Physical Medicine and Rehabilitation. Neurology. 2009b; 72(2):177-184. [PubMed: 19056667]

17. Notermans NC, Wokke JH, van der Graaf Y, Franssen H, van Dijk GW, Jennekens FG. Chronic idiopathic axonal polyneuropathy: a five year follow up. J Neurol Neurosurg Psychiatry. 1994b; 57(12):1525-1527. [PubMed: 7798984]

18. Periquet MI, Novak V, Collins MP, et al. Painful sensory neuropathy: prospective evaluation using skin biopsy. Neurology. 1999; 53(8):1641-1647. [PubMed: 10563606]

19. Hughes RA, Umapathi T, Gray IA, et al. A controlled investigation of the cause of chronic idiopathic axonal polyneuropathy. Brain. 2004; 127(Pt 8):1723-1730. [PubMed: 15201191]

20. Lindh J, Tondel M, Osterberg A, Vrethem M. Cryptogenic polyneuropathy: clinical and neurophysiological findings. J Peripher Nerv Syst. 2005; 10(1):31-37. [PubMed: 15703016]

21. Prineas J. Polyneuropathies of undetermined cause. Acta Neurol Scand Suppl. 1970; 44:3-72. [PubMed: 4325955]

22. Gorson KC, Ropper AH. Idiopathic distal small fiber neuropathy. Acta Neurol Scand. 1995; 92(5): 376-382. [PubMed: 8610490]

23. Barohn RJ. Approach to peripheral neuropathy and neuronopathy. Semin Neurol. 1998; 18(1):718. [PubMed: 9562663]

24. Saperstein DS, Wolfe GI, Gronseth GS, et al. Challenges in the identification of cobalamindeficiency polyneuropathy. Arch Neurol. 2003; 60(9):1296-1301. [PubMed: 12975298]

25. Allen RH, Stabler SP, Savage DG, Lindenbaum J. Diagnosis of cobalamin deficiency I: usefulness of serum methylmalonic acid and total homocysteine concentrations. Am J Hematol. 1990; 34(2): 90-98. [PubMed: 2339683] 
26. Savage DG, Lindenbaum J, Stabler SP, Allen RH. Sensitivity of serum methylmalonic acid and total homocysteine determinations for diagnosing cobalamin and folate deficiencies. Am J Med. 1994; 96(3):239-246. [PubMed: 8154512]

27. Kyle RA. 'Benign' monoclonal gammopathy. A misnomer. JAMA. 1984; 251(14):1849-1854. [PubMed: 6422066]

28. Bosch EP, Smith BE. Peripheral neuropathies associated with monoclonal proteins. Med Clin North Am. 1993; 77(1):125-139. [PubMed: 8380479]

29. Kissel JT, Mendell JR. Neuropathies associated with monoclonal gammopathies. Neuromuscul Disord. 1996; 6(1):3-18. [PubMed: 8845716]

30. Ropper AH, Gorson KC. Neuropathies associated with paraproteinemia. N Engl J Med. 1998; 338(22):1601-1607. [PubMed: 9603799]

31. Latov N. Pathogenesis and therapy of neuropathies associated with monoclonal gammopathies. Ann Neurol. 1995; 37(Suppl 1):S32-S42. [PubMed: 8968215]

32. Notermans NC, Wokke JH, Lokhorst HM, Franssen H, van der Graaf Y, Jennekens FG. Polyneuropathy associated with monoclonal gammopathy of undetermined significance. A prospective study of the prognostic value of clinical and laboratory abnormalities. Brain. 1994a; 117(Pt 6):1385-1393. [PubMed: 7820574]

33. Notermans NC, Wokke JH, van den Berg LH, et al. Chronic idiopathic axonal polyneuropathy. Comparison of patients with and without monoclonal gammopathy. Brain. 1996; 119(Pt 2):421427. [PubMed: 8800937]

34. Pestronk A, Li F, Griffin J, et al. Polyneuropathy syndromes associated with serum antibodies to sulfatide and myelin-associated glycoprotein. Neurology. 1991; 41(3):357-362. [PubMed: 1706491]

35. Nemni R, Fazio R, Quattrini A, Lorenzetti I, Mamoli D, Canal N. Antibodies to sulfatide and to chondroitin sulfate $\mathrm{C}$ in patients with chronic sensory neuropathy. J Neuroimmunol. 1993; 43(12):79-85. [PubMed: 8384638]

36. Wise CA, Garcia CA, Davis SN, et al. Molecular analyses of unrelated Charcot-Marie- Tooth (CMT) disease patients suggest a high frequency of the CMTIA duplication. Am J Hum Genet. 1993; 53(4):853-863. [PubMed: 8105684]

37. Nelis E, Van Broeckhoven C, De Jonghe P, et al. Estimation of the mutation frequencies in Charcot-Marie-Tooth disease type 1 and hereditary neuropathy with liability to pressure palsies: a European collaborative study. Eur J Hum Genet. 1996; 4(1):25-33. [PubMed: 8800924]

38. Leonardis L, Zidar J, Ekici A, Peterlin B, Rautenstrauss B. Autosomal dominant Charcot-MarieTooth disease type $1 \mathrm{~A}$ and hereditary neuropathy with liability to pressure palsies: detection of the recombination in Slovene patients and exclusion of the potentially recessive Thr118Met PMP22 point mutation. Int J Mol Med. 1998; 1(2):495-501. [PubMed: 9852256]

39. Choi BO, Lee MS, Shin SH, et al. Mutational analysis of PMP22, MPZ, GJB1, EGR2 and NEFL in Korean Charcot-Marie-Tooth neuropathy patients. Hum Mutat. 2004; 24(2):185-186. [PubMed: 15241803]

40. Schecht HM, Olney RK. Clinically sensory distal axonal polyneuropathy: early motor involvement. Electroencephalography and Clinical Neurophysiology. 1996 Mar; Volume 98(Number 3):26P-26P(1).

41. Vrancken AF, Franssen H, Wokke JH, Teunissen LL, Notermans NC. Chronic idiopathic axonal polyneuropathy and successful aging of the peripheral nervous system in elderly people. Arch Neurol. 2002 Apr; 59(4):533-540. [PubMed: 11939887]

42. Holland NR, Crawford TO, Hauer P, Cornblath DR, Griffin JW, McArthur JC. Small-fiber sensory neuropathies: clinical course and neuropathology of idiopathic cases. Ann Neurol. 1998; 44(1):4759. [PubMed: 9667592]

43. Gruener G, Dyck PJ. Quantitative sensory testing: methodology, applications, and future directions. J Clin Neurophysiol. 1994; 11(6):568-583. [PubMed: 7860720]

44. Finnerup NB, Johannesen IL, Fuglsang-Frederiksen A, Bach FW, Jensen TS. Sensory function in spinal cord injury patients with and without central pain. Brain. 2003; 126(Pt 1):57-70. [PubMed: 12477697] 
45. Freeman R, Chase KP, Risk MR. Quantitative sensory testing cannot differentiate simulated sensory loss from sensory neuropathy. Neurology. 2003; 60(3):465-470. [PubMed: 12578928]

46. Geber C, Klein T, Azad S, Birklein F, Gierthmühlen J, Huge V, Lauchart M, Nitzsche D, Stengel M, Valet M, Baron R, Maier C, Tölle T, Treede RD. Test-retest and interobserver reliability of quantitative sensory testing according to the protocol of the German Research Network on Neuropathic Pain (DFNS): a multi-centre study. Pain. 2011 Mar; 152(3):548-556. [PubMed: 21237569]

47. Shy ME, Frohman EM, So YT, Arezzo JC, Cornblath DR, Giuliani MJ, Kincaid JC, Ochoa JL, Parry GJ, Weimer LH. Therapeutics and Technology Assessment Subcommittee of the American Academy of Neurology. Quantitative sensory testing: report of the Therapeutics and Technology Assessment Subcommittee of the American Academy of Neurology. Neurology. 2003 Mar 25; 60(6):898-904. [PubMed: 12654951]

48. Chin RL, Latov N, Sander HW, et al. Sensory CIDP presenting as cryptogenic sensory polyneuropathy. J Peripher Nerv Syst. 2004; 9(3):132-137. [PubMed: 15363060]

49. McArthur JC, Stocks EA, Hauer P, Cornblath DR, Griffin JW. Epidermal nerve fiber density: normative reference range and diagnostic efficiency. Arch Neurol. 1998; 55(12):1513-1520. [PubMed: 9865794]

50. Devigili G, Tugnoli V, Penza P, et al. The diagnostic criteria for small fibre neuropathy: from symptoms to neuropathology. Brain. 2008; 131(Pt 7):1912-1925. [PubMed: 18524793]

51. Walk D, Wendelschafer-Crabb G, Davey C, Kennedy WR. Concordance between epidermal nerve fiber density and sensory examination in patients with symptoms of idiopathic small fiber neuropathy. J Neurol Sci. 2007; 255(1-2):23-26. [PubMed: 17337273]

52. Herrmann DN, Griffin JW, Hauer P, Cornblath DR, McArthur JC. Epidermal nerve fiber density and sural nerve morphometry in peripheral neuropathies. Neurology. 1999; 53(8):1634-1640. [PubMed: 10563605]

53. Lauria G, Cornblath DR, Johansson O, et al. EFNS guidelines on the use of skin biopsy in the diagnosis of peripheral neuropathy. Eur J Neurol. 2005; 12(10):747-758. [PubMed: 16190912]

54. Pasnoor M, Herbelin L, Johnson M, Ryals J, Dimachkie M, Wright D, Barohn R. Clinical electrophysiologic and skin biopsy findings in cryptogenic sensorimotor polyneuropathy compared to diabetic polyneuropathy and normal controls(abstract). Neurology. 2009; 72(Suppl 3):A217.

55. Pasnoor M, Nascimento O, Trivedi J, Wolfe GI, Nations S, Herbelin L, Freitas, Quintanilha G, Khan S, Dimachkie M, Barohn RJ. North America and South America (NA-SA) Neuropathy Project. Submitted to International Journal of Neuroscience.

56. Teunissen LL, Notermans NC, Franssen H, van der Graaf Y, Oey PL, Linssen WH, van Engelen BG, Ippel PF, van Dijk GW, Gabreëls-Festen AA, Wokke JH. Differences between hereditary motor and sensory neuropathy type 2 and chronic idiopathic axonal neuropathy. A clinical and electrophysiological study. Brain. 1997 Jun; 120(Pt 6):955-962. [PubMed: 9217680]

57. Mittal M, Pasnoor M, Mummaneni RB, Khan S, McVey A, Saperstein D, Herbelin L, Ridings L, Wang Y, Dimachkie MM, Barohn RJ. Retrospective chart review of duloxetine and pregabalin in the treatment of painful neuropathy. Int J Neurosci. 2011 Sep; 121(9):521-527. [PubMed: 21671841]

58. Attal N, Cruccu G, Haanpaa M, et al. EFNS guidelines on the pharmacological treatment of neuropathic pain. Eur J Neurol. 2006; 13(11):1153-1169. [PubMed: 17038030]

59. Dworkin RH, O'Connor AB, Backonja M, et al. Pharmacologic management of neuropathic pain: evidence-based recommendations. Pain. 2007; 132(3):237-251. [PubMed: 17920770]

60. Moulin DE, Clark AJ, Gilron I, et al. Pharmacological management of chronic neuropathic pain consensus statement and guidelines from the Canadian Pain Society. Pain Res Manag. 2007; 12(1):13-21. [PubMed: 17372630]

61. O'Connor AB, Dworkin RH. Treatment of neuropathic pain: an overview of recent guidelines. Am J Med. 2009; 122(10 Suppl):S22-S32. [PubMed: 19801049]

62. Gilron I, Bailey JM, Tu D, Holden RR, Weaver DF, Houlden RL. Morphine, gabapentin, or their combination for neuropathic pain. N Engl J Med. 2005; 352(13):1324-1334. [PubMed: 15800228]

63. Bril V, England J, Franklin GM, et al. Evidence-based guideline: treatment of painful diabetic peripheral neuropathy. Neurology. 2011; 76:1758-1765. [PubMed: 21482920] 
64. Slogosky SL, Chavin JM, Heiman-Patterson TT, ahmoush AJ. Idiopathic axonal neuropathy responsive to immunosuppression [abstract]. Ann Neurol. 1995:38336.

65. Donofrio PD, Berger A, Brannagan TH 3rd, et al. Consensus statement: the use of intravenous immunoglobulin in the treatment of neuromuscular conditions report of the AANEM ad hoc committee. Muscle Nerve. 2009; 40(5):890-900. [PubMed: 19768755]

66. Richardson JK. Factors associated with falls in older patients with diffuse polyneuropathy. J Am Geriatr Soc. 2002 Nov; 50(11):1767-1773. [PubMed: 12410893]

67. Tofthagen C, Visovsky C, Berry DL. Strength and balance training for adults with peripheral neuropathy and high risk of fall: current evidence and implications for future research. Oncol Nurs Forum. 2012 Sep; 39(5):E416-E424. [PubMed: 22940521] 


\section{KEY POINTS}

- Cyrptogenic or idiopathic sensory polyneuropathy is a common type of neuropathy seen in patients usually over age 50.

- Symptoms and signs are predominantly sensory while motor manifestations are usually mild or absent.

- Extensive evaluation usually does not reveal any identifiable abnormalities on laboratory testing. In up to one third of cases, electrophysiologic testing is normal. Skin biopsy may be helpful in this subgroup.

- Management is focused on patient reassurance, treatment of the neuropathic pain and rehabilitative care. 


\section{Table 1}

Studies of polyneuropathy patients with percentages of idiopathic cases.

\begin{tabular}{|l|c|c|}
\hline Authors & PN Patients & Idiopathic patients \\
\hline Prineas (1970) & 278 & $107(38 \%)$ \\
\hline Dyck et al. (1981) & 205 & $49(24 \%)$ \\
\hline Fagius (1983) & 91 & $67(74 \%)$ \\
\hline Konig et al. (1984) & 70 & $10(14 \%)$ \\
\hline McLeod et al. (1984) & 519 & $67(13 \%)$ \\
\hline Corvisier et al. (1987) & 432 & $48(11 \%)$ \\
\hline Notermans et al. (1993) & 500 & $75(10 \%)$ \\
\hline Wolfe et al (1999) & 402 & $93(23 \%)$ \\
\hline Jann et al (2001) & 222 & $48(21 \%)$ \\
\hline
\end{tabular}




\section{Table 2}

Total number of cases and diagnosis rate in six major categories.

\begin{tabular}{|l|l|l|}
\hline Major category & NA(No. of pts)(\%) & SA(No. of pts)(\%) \\
\hline Total no. of cases & 1090 & 1034 \\
\hline Immune mediated & $215(19.7 \%)$ & $191(18 \%)$ \\
\hline Diabetic & $148(13.5 \%)$ & $236(23 \%)$ \\
\hline Hereditary/degenerative & $292(26.7 \%)$ & $103(10 \%)$ \\
\hline Infect./inflamm. & $53(4.8 \%)$ & $141(14 \%)$ \\
\hline Syst./metab./toxic (Non-diabetic) & $71(6.5 \%)$ & $124(12 \%)$ \\
\hline Cryptogenic & $311(28.5 \%)$ & $239(23 \%)$ \\
\hline
\end{tabular}




\section{Table 3}

(adapted from Wolfe, 1999) ${ }^{2}$

Diagnostic Criteria for CSPN*

Inclusion Criteria

Symptoms

- Loss of sensation (numbness) or altered sensation (tingling/paresthesia/dysesthesia or pain beginning in the distal extremities(usually with onset in feet before hands)

- $\quad$ Symptoms present for at least 3 months

- $\quad$ No symptoms of weakness

- Symptoms of gait unsteadiness and autonomic dysfunction are allowable

Signs

- Sensory signs are present in a symmetrical fashion in distal limbs and may include any of the following: loss of vibration, proprioception, light touch, pain(pinprick), or temperature

- Hyporeflexia or areflexia may be present but is not required, even at the ankles

- Minimal weakness or atrophy is allowable in muscles supplying movement to the fingers and toes

Laboratory Studies

- $\quad$ Electrophysiology: sensory and motor NCS and needle EMG are often, but not invariably, abnormal; when abnormal, findings indicate a primarily axonal PN

- Quantitative sensory tests: vibration and temperature thresholds are often, but not invariably abnormal

- $\quad$ Other studies: if NCS/EMG and QST are normal, other studies including skin punch biopsy to measure epidermal nerve fiber density and autonomic studies including sudomotor tests (quantitative sudomotor axon reflex test, Silastic imprint testing, sympathetic skin response) and vasomotor test (heart rate variability to deep breathing, Valslva ratio) may provide evidence of peripheral nerve dysfunction

- $\quad$ Blood and urine tests: these should be normal or negative; a monoclonal protein by serum protein electrophoresis and/or immunofixation electrophoresis is allowable in patients with MGUS

\section{Exclusion criteria}

- Any identifiable metabolic, toxic, infectious, systemic, or hereditary disorder known to cause PN

- NCS abnormalities consistent with demyelination

- If a monoclonal gammopathy is present, the presence of an underlying lymphoproliferative disorder, malignancy, or amyloidosis

- Weakness on examination other than mild toes and/or finger weakness

*SPN indicates cryptogenic sensory polyneuropathy; NCS, nerve conduction studies; EMG, electromyography; PN; polyneuropathy, QST, quantitative sensory testing; and MGUS, monoclonal gammopathy of uncertain significance. 